\title{
The accuracy of cardiac MRI in differentiating between intra cardiac tumors and thrombi
}

\author{
Orly Goitein ${ }^{1 *}$, Einat Slonimasky ${ }^{1}$, Ashraf Hamdan ${ }^{2}$, Yishay Salem ${ }^{1}$, Elio Di Segni ${ }^{1}$, Osnat Konen $^{3}$, Eli Konen ${ }^{1}$ \\ From 18th Annual SCMR Scientific Sessions \\ Nice, France. 4-7 February 2015
}

\section{Background}

Primary or metastatic cardiac tumors are rare.

Echocardiography is limited in differentiating different cardiac masses in particular tumors from thrombi. MRI offers incremental value; optimal tissue differentiation especially with Gadolinium contrast enhancement. The ability to differntiate a cardiac tumor from a cardiac thrombus is crucial for patient management. The purposse of this study was to evaluate the accuracy of CMR in differentiating between intra cardiac tumors and thrombi.

\section{Methods}

Retrospective analysis of a prospectively maintained database (2004-2013) was performed. This included all patients referred for the evaluation of intra-cardiac tumors versus thrombi. CMR sequences included: T2, gradient echo,T1 before and after Gadolinium (Gd) administration, first pass perfusion and delayed enhancement. CMR findings were compared to the definitive diagnosis of the intra cardiac - mass established either by pathology and /or clinical and echocardiographic follow up, when available. Accuracy, sensitivity and positive predictive volume (PPV) of CMR for differentiating between tumor and thrombus were calculated, accordingly.

\section{Results}

Of the 150 patients referred for CMR, intra cardiac masses were detected at in 111 patients. At CMR cardiac tumors were diagnosed in 72 patients, thrombi in 28 patients ; 11 masses were inconclusive. Definitive diagnosis was available in 66 patients including 49 tumors and 17 thrombi. CMR correctly diagnosed 48 out of 49 tumors ; sensitivity PPV and NPV were $97 \%, 97 \%$ and $92 \%$, respectively. Thrombi were correctly diagnosed at CMR in 14 out of 17 scans; sensitivity PPV and NPV were $82 \%, 93 \%$ and $80 \%$, respectively. The overall accuracy in differentiating intra-cardiac tumors from thrombi was $94 \%$. Of the 11 inconclusive lesions detected, 5 were smaller than $1 \mathrm{~cm}$.

\section{Conclusions}

The different CMR sequences allows reliable tissue characterization, thus enabling an accurate differentiation between intra cardiac tumors and thrombi. This high diagnostic potential is limited in lesion smaller than $1 \mathrm{~cm}$.

\section{Funding}

No external funding was used for this study.

\section{Authors' details}

${ }^{1}$ Diagnostic Imaging, Sheba Medical Center, Tel Hashomer, Israel. ${ }^{2}$ Heart Center, Sheba Medical Center, Tel Hashomer, Israel. 'Diagnostic Imaging, Schneider Children's Medical Center of Israel, Petach Tiquva, Israel.

Published: 3 February 2015

doi:10.1186/1532-429X-17-S1-Q70

Cite this article as: Goitein et al:: The accuracy of cardiac MRI in differentiating between intra cardiac tumors and thrombi. Journal of Cardiovascular Magnetic Resonance 2015 17(Suppl 1):Q70. 